phys. stat. sol. (b) 144, 73 (1987)

Subject classification: 61.30 and 61.40

Institut für Theoretische und Angewandte Physik der Universität Stuttgart ${ }^{1}$ )

\title{
Metallic Glasses and Chiral Nematic Liquid Crystals
}

By

\author{
H.-R. Trebin, L. Longa ${ }^{2}$ ), and B. SAlzgeber \\ Dedicated to Prof. Dr. A. SeEgrer on the occasion of his 60th birthday
}

Geometrical frustration exists in both metallic glasses and chiral nematic liquid crystals. Applying results from the Landau-Ginzburg theory of liquid crystals and from polytope models for the amorphous state, a frustrated free-energy density for metallic glasses is derived. It is expressed in terms of an order parameter that characterizes the bond orientation of neighbouring atoms.

Sowohl in metallischen Gläsern als auch in chiralen nematischen Flüssigkristallen gibt es geometrische Frustration. Wir benutzen die Landau-Ginzburg-Theorie für Flüssigkristalle und Polytop-Modelle für den amorphen Zustand um für metallische Gläser eine frustrierte Dichte der freien Energie abzuleiten. Der darin enthaltene Ordnungsparameter beschreibt die Bindungsorientierung benachbarter Atome.

\section{Introduction}

$\mathrm{X}$-ray structure analysis allows to specify the atomic and molecular positions of most complicated systems in physics, chemistry, and biology. Unit cells of crystals, for example, can be surveyed with high precision, even if they contain hundreds of atoms.

Nonetheless, in the past few years materials of unusual properties have been synthesized, whose atomic and molecular setup is not yet completely unveiled despite of worldwide intensive efforts. Of these, we pick out two, which at first sight appear as very different, but whose structures are closely related by basic geometrical concepts; these are metallic glasses and chiral nematic liquid crystals.

The structural models for metals - either monatomic or as alloys - conceive the atoms as almost hard spheres, which tend to pack as densely as possible [1]. There are hints $[2,3]$ that locally the spheres attempt to arrange themselves into icosahedra, twelve of them surrounding a central one. Globally, there are roughly speaking five phases: the melt, the metallic glass, the icosahedral phase, the T-phase, and the crystalline state.

In many instances, like the Frank-Kasper alloys [4], the crystalline phases possess a large unit cell containing many icosahedra. In the lattices one can identify periodic arrays of lines, which are interpreted as wedge disclinations of an icosahedral state [5].

For the pure icosahedral phase [6] there are several models, the most spectacular being that of a three-dimensional Penrose pattern [ 7 to 9].

The ' $\mathrm{T}$-phase $[10,11]$ is periodic along only one direction in space. The order in the planes perpendicular to the axis of periodicity is assumed to be closely related to a two-dimensional Penrose pattern. The atomic positions in both the icosahedral phase and the T-phase are still a subject of dispute.

1) Pfaffenwaldring 57, D-7000 Stuttgart 80, FRG.

2) Alexander-von-Humboldt Foundation Fellow 1985 to 87, Permanent address: Department of Statistical Physics, Jagellonian University, Reymonta 4, Krakow, Poland. 
The metallic glass is amorphous. A commonly accepted model, pointing out its characteristics, is still missing, as well as an explanation of its universal features, like the extra heat capacity proportional to $T$ at low temperatures, or the Vogel-Fulcher law for the temperature dependence of the transport coefficients [12].

Chiral nematic liquid crystals are liquids, consisting of elongated molecules, which lack a centre of inversion [13]. At low temperatures, the molecular axes align along a preferred direction in space, which is marked by a unit vector $\hat{\boldsymbol{n}}$, the director. The director field, however, is not uniform. It twists along an axis perpendicular to the director. The result is an orientational pattern, the cholesteric phase, which like the T-phase displays a discrete translational symmetry along one spatial direction. The repeat distance (pitch) is of the order of $10^{2}$ to $10^{3} \mathrm{~nm}$.

If the temperature is raised, then in a temperature interval of about $1 \mathrm{~K}$ two phases of cubic space group are found [14], with a lattice parameter of the order of the cholesteric pitch. Because of the large size of the unit cell they reflect light preferably in the short-wavelength part of the visible spectrum and, therefore, are denoted Blue Phases I and II. They are again purely orientational patterns of the molecular axes, the centres of mass of the molecules moving as in a liquid. Although the cubic space groups of the patterns are well established, the detailed pattern in the unit cell is unconfirmed. Several groups assume that these anisotropic liquids are traversed by cubic arrays of disclination lines [15 to 17].

In the same narrow temperature interval, slightly above the cubic Blue Phases and before the isotropic phase is reached, a third phase exists, denoted Blue Fog or Blue Phase III [18, 19]. It appears structureless, but several groups interpret the Blue Fog as an orientational pattern of distorted icosahedral symmetry [20, 21].

Evidently, the phase structure of both materials is very similar. The parallelism becomes intelligible by the observation that in both systems the phenomenon of frustration is present in the following sense: the order, for which the energy becomes locally minimal, cannot be extended globally [22]. In the case of metals the icosahedral close-packing cannot be incorporated into a space-filling structure. In chiral nematic liquid crystals, frustration is read off a phenomenological free-energy density.

Using the analogies of both systems and well-known results from the Landau-Ginzburg theory of chiral nematic liquid crystals, we derive a frustrated continuum model for the free-energy density of metals. The model might serve for a unified description of most of the metallic phases.

\section{Chiral Nematic Liquid Crystals}

\subsection{Order parameter}

The orientational order in a small volume element of a chiral nematic liquid crystal is expressed by an angular distribution function $f(\hat{\boldsymbol{v}})$. The value $f(\hat{\boldsymbol{v}}) \mathrm{d} \Omega$ is the probability per unit volume that the long axis of a molecule points along the unit vector $\hat{v}$ into the solid angle $\mathrm{d} \Omega$. From experimental evidence one knows that the distribution function is of even parity: $f(\hat{\boldsymbol{v}})=f(-\hat{\boldsymbol{v}})$. Therefore, in lowest order of a multipole expansion, the deviation from isotropy is described by a quadrupole term $\hat{\boldsymbol{v}} \cdot Q \hat{\boldsymbol{v}}$, where $Q$ denotes a traceless, symmetric, second-rank tensor. The quadrupole tensor $Q$ serves as an order parameter. In a non-uniform liquid crystal it depends on position : $Q=Q(x)$.

\subsection{Free-energy density}

According to the Landau-Ginzburg theory of phase transitions [13], the free-energy density of chiral nematic liquid crystals is expanded into $\mathrm{SO}(3)$-invariant polynomials in the components of $Q=\left[Q_{i j}\right]$ and its gradient $\nabla Q=\left[Q_{i j, k}=\left(\partial / \partial x_{k}\right) Q_{i j}=\partial_{k} Q_{i j}\right]$. 
$\mathrm{O}(3)$-invariance cannot be claimed, because the liquid is optically active also in the isotropic phase and is lacking mirror symmetry.

The invariant expansion is divided into a bulk term and an elastic term,

$$
F=F_{\text {bulk }}+F_{\mathrm{el}},
$$

where $F_{\text {bulk }}$ usually is truncated after the fourth order in $Q$,

$$
F_{\text {bulk }}=\frac{1}{2} A \operatorname{tr} Q^{2}-\frac{1}{3} B \operatorname{tr} Q^{3}+\frac{1}{4} C\left(\operatorname{tr} Q^{2}\right)^{2},
$$

and $F_{\text {el }}$ after the second order in $\nabla Q$,

$$
F_{\mathrm{el}}=\frac{1}{2} L_{1} Q_{i j, k} Q_{i j, k}+\frac{1}{2} L_{2} Q_{i j, j} Q_{i k, k}+\frac{1}{2} L_{3} Q_{i j, k} Q_{i k, j}+\frac{1}{2} L_{4} \varepsilon_{i k j} Q_{i l} Q_{j l, k}
$$

with elastic constants $L_{r}$. Summation over repeated indices is assumed implicitly.

The last term of (3) is denoted chiral, because it is linear in the gradient and breaks $\mathrm{O}(3)$-symmetry to $\mathrm{SO}(3)$. Unique features of $F_{\text {el }}$ come to light, if to the nonchiral part of $F_{\text {el }}$ we apply what is called the "one-constant approximation". It amounts to neglecting the elastic constants $L_{2}$ and $L_{3}$ compared to $L_{1}$. The assumption is roughly justified by experiment, which yields the ratios $L_{2} / L_{1}, L_{3} / L_{1}$ to about 10 to $30 \%$. The one-constant form of $F_{\text {el }}$ also comes out if invariance is postulated under independent rotations in position space $R^{3}$ and in order-parameter space $\{Q\}(\mathrm{SO}(3) \times \mathrm{SO}(3)$ symmetry).

Using the notations $L=L_{1}, q=L_{4} / 4 L_{1}$, we can press $F_{\mathrm{el}}$ into the following compact form:

$$
F_{\mathrm{el}}=\frac{1}{2} L\left\{\left(D_{k} Q\right)_{i j}\left(D_{k} Q\right)_{i j}-6 q^{2} \operatorname{tr} Q^{2}\right\} .
$$

The operator $D$ is defined by

$$
\left(D_{k} Q\right)_{i j}=\partial_{k} Q_{i j}+q \varepsilon_{k i l} Q_{l j}+q \varepsilon_{k j l} Q_{l i} .
$$

Equations (4) and (5) become more transparent, if we represent $Q$ in polar coordinates $Q_{m}^{(2)}, m=-2,-1, \ldots, 2[23]$. Then (4) turns to

where

$$
F_{\mathrm{el}}=\frac{1}{2} L\left\{\left(D_{k} Q^{(2)}\right)_{m}^{*}\left(D_{k} Q^{(2)}\right)_{m}-6 q^{2}\left|Q^{(2)}\right|^{2}\right\},
$$

and

$$
\left|Q^{(2)}\right|^{2}=Q_{m}^{(2) *} Q_{m}^{(2)}
$$

$$
D_{k} Q^{(2)}=\left(\partial_{k}-i q J_{k}\right) Q^{(2)}
$$

with components

$$
\begin{aligned}
& \left(D_{k} Q^{(2)}\right)_{m}=\left(\delta_{m m^{\prime}} \partial_{k}-i q J_{k m m^{\prime}}\right) Q_{m^{\prime}}^{(2)} \\
& J_{k m m^{\prime}}=\left\langle l m\left|J_{k}\right| l m^{\prime}\right\rangle
\end{aligned}
$$

denote the matrix elements of the angular momentum operators $J_{1}, J_{2}, J_{3}$ in a standard basis with quantum number $l=2$.

\subsection{Reduced order parameter}

The weight of the elastic part of the free-energy density in comparison to the bulk part is judged by the coherence length $\xi=\sqrt{|A| / L}$. If the typical length scale of the spatial variation of $Q(x)$ is much larger than $\xi$, then $F_{\text {bulk }}$ dominates $F_{\text {el }}$, and it is legitimate to divide the minimization process of $F$ into two steps, viz. first of $F_{\text {bulk }}$, then of $F_{\text {el }}$.

The quadratic and quartic terms of $F_{\text {bulk }}$ form a polynomial in $\operatorname{tr} Q^{2}$ and fix the equilibrium value of the modulus $S=\sqrt{\operatorname{tr} Q^{2}}=\left|Q^{(2)}\right|$ of the order parameter. The cubic term, $-\frac{1}{2} B \operatorname{tr} Q^{3}$, is reponsible for two of the eigenvalues of $Q$ being degenerate in equilibrium [24]. Thus $Q$ is of cylinder symmetry with respect to the nondegenerate eigenspace, which is represented by the director $\hat{\boldsymbol{n}}$, and the tensors minimizing $\boldsymbol{F}_{\text {bulk }}$ 
(the "vacuum") are written as

$$
Q=\sqrt{\frac{3}{2}} S\left\{\hat{\boldsymbol{n}} \otimes \hat{\boldsymbol{n}}-\frac{1}{3} I\right\} .
$$

$I$ denotes the unit tensor. The director is the only remaining degree of freedom and is called "reduced order parameter".

In this ("hydrodynamic") limit the bulk term is a constant, and the elastic term merely depends on the spatial variations of $\hat{\boldsymbol{n}}(\boldsymbol{x})$. If under these conditions (11) is inserted into (3), the elastic free energy becomes a polynomial in the components $n_{i, j}$ of $\nabla \hat{\boldsymbol{n}}$

$$
F_{\mathrm{el}}=\frac{1}{2}\left(3 S^{2} L\right)\left\{\left(D_{k} \hat{\boldsymbol{n}}\right) \cdot\left(D_{k} \hat{\boldsymbol{n}}\right)-2 q^{2}\right\} .
$$

The operator $D$ is defined by

or

$$
\left(D_{k} \hat{\boldsymbol{n}}\right)_{j}=\partial_{k} n_{j}+q \varepsilon_{k j l} n_{l}
$$

$$
D \hat{\boldsymbol{n}}=\left(\partial_{k}-i q L_{k}\right) \hat{\boldsymbol{n}} \text {. }
$$

The operator $i L_{k}$ of components

$$
\left(L_{k}\right)_{i j}=i \varepsilon_{k i j}
$$

generates the rotations of three-dimensional vectors about the axis $k$.

\subsection{Frustration}

The single spiral

$$
\hat{\boldsymbol{n}}(\boldsymbol{x})=\mathrm{e}^{i q L_{3} z} \hat{\boldsymbol{e}}_{\mathbf{1}}=\hat{\boldsymbol{e}}_{\mathbf{1}} \cos q z-\hat{\boldsymbol{e}}_{\mathbf{2}} \sin q z
$$

along the $z$-axis yields a free-energy density

$$
F_{\mathrm{el}}=-\frac{3}{2} L S^{2} q^{2}
$$

A lower value, viz.

$$
F_{\mathrm{el}}=-2 L S^{2} q^{2}
$$

is obtained, if we insert into (4) or (6) the single biaxial spiral

$$
Q^{(2)}(x)=\frac{S}{\sqrt{2}} \mathrm{e}^{i q J_{3} z}\{|22\rangle+|2-2\rangle\},
$$

where $|2 \pm 2\rangle$ denotes pure $m= \pm 2$-states of components $Q_{m}^{(2)}=\delta_{m, \pm 2}$, respectively. In Cartesian components, (19) reads

$$
Q(\boldsymbol{x})=\frac{S}{\sqrt{2}}\left\{\left(\hat{\boldsymbol{e}}_{\mathbf{1}} \otimes \hat{\boldsymbol{e}}_{1}-\hat{\boldsymbol{e}}_{\mathbf{2}} \otimes \hat{\boldsymbol{e}}_{2}\right) \cos 2 q z+\left(\hat{\boldsymbol{e}}_{\mathbf{1}} \otimes \hat{\boldsymbol{e}}_{\mathbf{2}}+\hat{\boldsymbol{e}}_{\mathbf{2}} \otimes \hat{\boldsymbol{e}}_{\mathbf{1}}\right) \sin 2 q z\right\} .
$$

Tensor (20) has eigenvalues $S / \sqrt{2}, 0,-S / \sqrt{2}$, is maximal biaxial, and of $\operatorname{tr} Q^{3}=0$. Although $F_{\text {el }}$ is low, the cubic bulk term is not minimal: we note a competition between bulk and elastic terms.

The absolute minimum of the elastic energy

$$
F_{\mathrm{el}}=-3 L S^{2} q^{2}
$$

is obtained, if the positive definite terms in (4) and (12) vanish, which is the case for $D_{k} Q=0$ and $D_{k} \hat{\boldsymbol{n}}=0$. Since the bulk energy is minimized by an uniaxial order parameter, the true ground state is of form (11), where the director solves

$$
\partial_{k} \hat{\boldsymbol{n}}=i q L_{k} \hat{\boldsymbol{n}} ; \quad k=\mathbf{1}, \mathbf{2}, \mathbf{3}
$$


everywhere in space. Integrating (22) we find that in proceeding form $x$ to $x+\Delta x$,

$$
\hat{\boldsymbol{n}}(\boldsymbol{x}+\Delta \boldsymbol{x})=\mathrm{e}^{i q \Delta \boldsymbol{x} \cdot \boldsymbol{L}} \hat{\boldsymbol{n}}(\boldsymbol{x}),
$$

i.e. $\hat{\boldsymbol{n}}$ is rotated by an angle $|q \Delta \boldsymbol{x}|$ about axis $\widehat{\Delta \boldsymbol{x}}$. The director field must form a multiple twist.

Condition (23) can be met for all points $\boldsymbol{x}$ on a line, but not on a plane. If one encircles a square, twisting the director in accord with (23), in general one does not return to the starting director. There is no director field which at each point of a finite volume assumes the minimum value of the free-energy density: the system is geometrically frustrated.

\subsection{Relieve of frustration on the three-sphere}

Sethna et al. [22] have demonstrated that on a three-sphere of radius $R=1 / q$ (which bounds a ball in four-dimensional space) the frustration is relieved. When forming a free-energy density on $\mathrm{S}^{3}$, one has to replace the derivative operator $\partial_{k}$ in (13) and (14) by the canonical covariant derivative of the Levi-Civita connection on $\mathrm{S}^{3}$. In a standard basis $\left\{\hat{\boldsymbol{e}}_{1}(p), \hat{\boldsymbol{e}}_{2}(p), \hat{\boldsymbol{e}}_{3}(p) \mid p \in \mathrm{S}^{3}\right\}$, which is introduced below, the substitution is

$$
\partial_{k} n_{j} \quad \text { by } \partial_{k} n_{j}-q \varepsilon_{k j l} n_{l},
$$

the second term just compensating the angular momentum operator $i q L_{k}$ in (14). Then, on $\mathrm{S}^{3}$ the elastic free-energy density reads

$$
F_{\text {el }}=\frac{1}{2}\left(3 \mathrm{~S}^{2} L\right)\left\{\left(\partial_{k} n_{j}\right)\left(\partial_{k} n_{\jmath}\right)-2 q^{2}\right\},
$$

and is minimized by any director field of constant components $n_{j}$ in the basis $\left\{\hat{\boldsymbol{e}}_{k}(p)\right\}$.

The three-sphere $\mathrm{S}^{3}$ is isomorphic to the Lie group $\mathrm{SU}(2)$ of unitary complex $2 \times 2$ matrices via the correspondence of a point

$$
R\left[a_{0}, a_{1}, a_{2}, a_{3}\right]
$$

of $\mathrm{S}^{3}$ with a point

$$
a_{0} 1+a_{1} i \dot{\sigma}_{1}+a_{2} i \sigma_{2}+a_{3} i \sigma_{3}
$$

of $\mathrm{SU}(2)$. The $\sigma_{k}$ are Pauli matrices, 1 is the unit $2 \times 2$ matrix, and the components $a$ are normalized to $1, a_{0}^{2}+a_{1}^{2}+a_{2}^{2}+a_{3}^{2}=1$.

The tangent space at the northpole $[\mathrm{R}, 0,0,0]$ is spanned by basis vectors $[0,1,0,0]$, $[0,0,1,0]$, and $[0,0,0,1]$. These correspond to the generators $i \sigma_{1}, i \sigma_{2}, i \sigma_{3}$ of the Lie algebra of $\mathrm{SU}(2)$. The standard basis at an element $g \in \mathrm{SU}(2)$ is the right translated triple $i \sigma_{1} g$, $i \sigma_{2} g$, $i \sigma_{3} g$. Any vector field $v(g)$ of constant components is generated by a vector $v(e)$ of the space tangent to the unit element (which corresponds to the northpole) by right translation to all points of $\mathrm{SU}(2)=\mathrm{S}^{3}: v(g)=v(e) g$.

If one rolls $\mathrm{S}^{3}$ on the flat three-dimensional space tangent to the northpole along the line connecting the origin with point $\boldsymbol{x}$, one obtains vectors

$$
\hat{\boldsymbol{n}}(\boldsymbol{x})=\mathrm{e}^{i q \boldsymbol{x} \cdot \boldsymbol{L}} \hat{\boldsymbol{n}}(0)
$$

satisfying the twisting condition (23).

Nature, being stuck to flat three-space, deals with frustration by growing complex orientational patterns with singularities - the Blue Phases. For the minimization of the free-energy density in flat space, which ultimately leads to these patterns [16, 17], the geometrical interpretation of frustration is of little help. However, it does aid in forming phenomenological frustrated free-energy densities for other systems. 
We have arrived at the idea of chiral nematic liquid crystals on $\mathrm{S}^{3}$ starting from an invariant expansion of the free-energy density. For the case of metals, we start from the geometrical model of the ideal metal on $\mathrm{S}^{3}$ and - going the way back - arrive at a frustrated free energy in flat space.

\section{Metallic Glasses}

\subsection{Polytope model}

The simplest assumption for the cohesive behaviour of metal atoms is that they are balls which at contact repel strongly and at large distances attract slightly. Accordingly, amorphous metals frequently are modelled by ensembles of densely packed balls [25]. In the plane, discs are packed most densely in an hexagonal array. Reviving ideas of Coxeter [26], Sadoc et al. [27], and Nelson [5] have pointed out that densest packing of three-dimensional balls is possible only in a eurved space, namely if their centres form the vertices of the polytope $\{3,3,5\}$. Polytopes are four-dimensional analogues of regular polyhedra [26]. Their vertices are located on the surface of a fourdimensional ball, which is the three-sphere $\mathrm{S}^{3}$, representing a three-dimensional curved manifold. On $\{3,3,5\}, 120$ atoms are arranged in 600 regular tetrahedra (Schläfli symbol $\{3,3\}$, five sharing an edge. Each atom is surrounded by twelve neighbours in an icosahedral array. If we use the isomorphism of $\mathrm{S}^{3}$ and $\mathrm{SU}(2)$, the 120 atomic centres on $\mathrm{S}^{3}$ correspond to the 120 elements of the double group $\overline{\mathrm{Y}}$, which is the lift of the 60 -elements icosahedral group $\mathrm{Y}$ c SO(3) into $\mathrm{SU}(2)$.

There is evidence that icosahedral neighbourhoods are present also in flat-space metals. Frank [2] noticed that 13 atoms, interacting via a Lennard-Jones potential, have an $8.4 \%$ larger binding energy in an icosahedral cluster than in clusters as found in f.c.c. or h.c.p. lattices. He assumed that icosahedral clusters abound in supercooled liquid metals. Steinhardt et al. [3] confirmed predominant icosahedral order in molecular-dynamics simulations of a supercooled Lennard-Jones liquid. When icosahedral quasicrystalline alloys are shot amorphous by electron irradiation, the decagonal stars of Bragg reflections smear out into rings of the same radius, strongly indicating that the local icosahedral neighbourhood persists [28].

But the icosahedral local order is of noncrystallographic symmetry and, like the chiral nematic multiple twist, cannot extend globally. In both cases the curvature of the three-sphere $\mathrm{S}^{3}$ is root and expression for frustration.

\subsection{Order parameter}

Bond-orientational order is characterized by an angular distribution function $f(\hat{\boldsymbol{v}})$, where $f(\hat{\boldsymbol{v}}) \mathrm{d} \Omega$ is the probability per unit volume for a bond to point into the solid angle $\mathrm{d} \Omega$ along the unit vector $\hat{\boldsymbol{v}}$. In a multipole expansion

$$
f(\hat{\boldsymbol{v}}, \boldsymbol{x})=\sum_{l=0}^{\infty} \sum_{m=-l}^{+l} Q_{m}^{(l)}(\boldsymbol{x}) \mathbf{Y}_{m}^{(l)}(\hat{\boldsymbol{v}})
$$

the first moment which can express faithful icosahedral symmetry is the one of $l=6$. Therefore, in [3] the irreducible tensor $Q^{(6)}$ has been used as order parameter for metallic glasses in an expansion of a bulk free-energy density up to cubic terms. Quartic terms have been added by Jarić [29] and Salzgeber [30] to yield

$$
\begin{aligned}
F_{\text {bulk }}= & \frac{1}{2} A\left|Q^{(6)}\right|^{2}-\frac{1}{3} B\left(\begin{array}{ccc}
6 & 6 & 6 \\
m_{1} & m_{2} & m_{3}
\end{array}\right) Q_{m_{1}}^{(6)} Q_{m_{2}}^{(6)} Q_{m_{3}}^{(6)}+ \\
& +\frac{1}{4} C_{0}\left|Q^{(6)}\right|^{4}+\frac{1}{4} C_{1} I_{6}^{4,1}+\frac{1}{4} C_{2} I_{6}^{4,2},
\end{aligned}
$$


where $I_{6}^{4,1}$ and $I_{6}^{4,2}$ are two nontrivial quartic SO(3)-invariants,

$$
\begin{aligned}
I_{6}^{4,1}= & (-1)^{n}\left(\begin{array}{ccc}
6 & 6 & 6 \\
m_{1} & m_{2} & n
\end{array}\right)\left(\begin{array}{ccc}
6 & 6 & 6 \\
-n & m_{3} & m_{4}
\end{array}\right) Q_{m_{1}}^{(6)} Q_{m_{2}}^{(6)} Q_{m_{3}}^{(6)} Q_{m_{4}}^{(6)}, \\
I_{6}^{4,2}= & (-1)^{n_{1}+n_{2}+n_{3}+n_{4}}\left(\begin{array}{ccc}
6 & 6 & 6 \\
-n_{1} & m_{1} & n_{2}
\end{array}\right)\left(\begin{array}{ccc}
6 & 6 & 6 \\
-n_{2} & m_{2} & n_{3}
\end{array}\right)\left(\begin{array}{ccc}
6 & 6 & 6 \\
-n_{3} & m_{3} & n_{4}
\end{array}\right) \times \\
& \times\left(\begin{array}{ccc}
6 & 6 & 6 \\
-n_{4} & m_{4} & n_{1}
\end{array}\right) Q_{m_{1}}^{(6)} Q_{m_{2}}^{(6)} Q_{m_{3}}^{(6)} Q_{m_{4}}^{(6)} .
\end{aligned}
$$

A systematic way to establish $\mathrm{SO}(3)$-invariants to all orders is provided by the theory of invariants (see e.g. [31 to 33]), and a corresponding analysis is performed in the Appendix.

Points in the 13-dimensional space of sixth-rank irreducible tensors can have the following symmetry groups [34]: $\mathrm{SO}(3), \mathrm{D}_{\infty}, \mathrm{Y}, \mathrm{O}, \mathrm{T}, \mathrm{D}_{6}, \mathrm{D}_{5}, \mathrm{D}_{4}, \mathrm{D}_{3}, \mathrm{D}_{2}, \mathrm{C}_{3}, \mathrm{C}_{2}, \mathrm{C}_{1}$. Jaric [29] has marked each point in parameter space $\left\{A, B, C_{0}, C_{1}, C_{2}\right\}$ of the bulk-freeenergy density (29) with the symmetry group of the corresponding vacuum tensors.

\subsection{Frustrated elastic free-energy density}

In the following we assume a parameter set to be chosen in (29) such that the tensors minimizing $F_{\text {bulk }}$ have icosahedral symmetry. Then, in the hydrodynamic limit, the full order parameter space $\left\{Q^{(6)}\right\}$ can be reduced to the set of orientations of a twelve-pronged star of vectors, which point from the centre of an icosahedron to the vertices. This set is isomorphic to the coset space $\mathrm{SO}(3) / \mathrm{Y}[35]$.

At each vertex of polytope $\{3,3,5\}$ the star is composed by the vectors pointing to the nearest neighbours. The reduced order parameter at the northpole e $\in \mathbf{S U}(2)==\mathrm{S}^{3}$ is the set of twelve elements of the Lie algebra of $\mathrm{SU}(2)$,

$$
\hat{\boldsymbol{n}}^{k}=n_{j}^{k} i \sigma_{j} ; \quad k=1, \ldots, 12 .
$$

The triples of components are

$$
\begin{aligned}
k & =1: & {\left[n_{j}^{1}\right] } & =[0,0,1], \\
k & =2, \ldots, 6: & {\left[n_{j}^{k}\right] } & =\left[\frac{2}{\sqrt{5}} \cos \frac{2 \pi(k-2)}{5}, \frac{2}{\sqrt{5}} \sin \frac{2 \pi(k-2)}{5}, \frac{1}{\sqrt{5}}\right], \\
k & =7, \ldots, 11: & {\left[n_{j}^{k}\right] } & =\left[-n_{j}^{k-5}\right], \\
k & =12: & {\left[n_{j}^{12}\right] } & =\left[-n_{j}^{1}\right] .
\end{aligned}
$$

It is straightforward to prove that the star at any other vertex $g \in \overrightarrow{\mathrm{Y}}$ of $\{3,3,5\}$ results from right translation $\widehat{\boldsymbol{n}}^{k} g$ of the twelve vectors (33).

This fact provides the recipe of how to extend the reduced order parameter field from the points of $\bar{Y}$ to all of SU (2) and to establish a continuum model for the bond orientation of the ideal metallic glass on $\mathrm{S}^{3}$ : we simply form twelve right-invariant vector fields $\hat{\boldsymbol{n}}^{k} g, g \in \mathrm{SU}(2)$. One may call this model "icosahedral chiral nematic phase", because it follows from the uniaxial chiral nematic phase by addition of eleven more vector fields.

The corresponding sixth-rank tensor field of icosahedral shape has components

$$
Q_{m}^{(6)}=\frac{S}{5}[0, i \sqrt{7}, 0,0,0,0, \sqrt{11}, 0,0,0,0,-i \sqrt{7}, 0]
$$

in the basis $\left(i \sigma_{j}\right) g$ at $g \in \mathrm{SU}(2)$. 
The vertices of $\{3,3,5\}$ and the order parameter field can be transferred consistently from $\mathrm{S}^{3}$ to flat space only along lines, by rolling. For example, if we roll $\mathrm{S}^{3}$ along a great circle connecting neighbouring vertices, $V_{1}, V_{2}, \ldots$, then in flat space we obtain a chain of slightly distorted intertwined icosahedra $I_{k}$, centred about the imprints $\tilde{V}_{k}$ of $V_{k} . \tilde{V}_{k+1}$ and $\tilde{V}_{k-1}$ are opposite points on $I_{k}$. $I_{k+1}$ results from $I_{k}$ by a right screw translation from $\tilde{V}_{k}$ to $\tilde{V}_{k+1}$ with screw angle $\pi / 5$ [30].

When we roll $\mathrm{S}^{3}$ in the tangent space from point 0 to $\boldsymbol{x}$, then the icosahedral sixthrank tensor field behaves like

$$
Q^{(6)}(x)=\mathrm{e}^{i q x \cdot J} Q^{(6)}(0) .
$$

$J_{1}, J_{2}, J_{3}$ are angular momentum operators acting in the space $\left\{Q^{(6)}\right\}, q$ is the inverse radius $1 / R$ of $\mathrm{S}^{3}$, where $R$ is the golden mean $\tau=\frac{1}{2}(1+\sqrt{5})$ times the diameter of the metal atoms.

Tensor field (35), which represents the ideal bond orientation, satisfies the differential equation

$$
\partial_{k} Q^{(6)}=i q J_{k} Q^{(6)} ; \quad k=1,2,3,
$$

at the origin. It is, therefore, reasonable to formulate a frustrated elastic energy analogous to $(6)$

$$
F_{\mathrm{el}}=\frac{1}{2} L\left(D_{k} Q^{(6)}\right)_{m}^{*}\left(D_{k} Q^{(6)}\right)_{m},
$$

where we incorporate the term proportional to $\left|Q^{(8)}\right|^{2}$ in the quadratic part of $F_{\text {bulk }}(29)$.

Equation (37) defines a frustrated free-energy density for metallic glasses in the oneconstant approximation. The main insight we gain from (37) is that a chiral term

$$
-i\left(\partial_{k} Q^{(6)}\right)_{m}^{*}\left(J_{k} Q^{(6)}\right)_{m}+\text { c.c. }
$$

linear in the gradient of $Q^{(6)}$ is necessary for expressing frustration.

The sign of the chirality, i.e. the sign of $q$ and the handedness of the screw, is an artefact of the continuum model. The twelve-pronged star at a point $g \in \overline{\mathrm{Y}}$ can also be obtained by a left translation $g \hat{\boldsymbol{n}}^{k}$ from the polar star (apart from a permutation of the numbering indices), and the icosahedron $I_{k+1}$ in flat space by a left screw translation from $I_{k}$. The sense of the screw rotation only becomes visible in the interpolation.

\subsection{Exact elastic free-energy density}

When going beyond the one-constant approximation, we must construct the elastic free-energy density as an $\mathrm{SO}(3)$-invariant polynomial in the components of $Q^{(6)}$ and its gradient.

For the invariant expansion we apply angular momentum calculus. We introduce spherical components for the derivative operator,

$$
\begin{aligned}
& \partial_{1}=-\frac{1}{\sqrt{2}}\left(\partial_{x}-i \partial_{y}\right), \\
& \partial_{0}=\partial_{z}, \\
& \partial_{-1}=\frac{1}{\sqrt{2}}\left(\partial_{x}+i \partial_{y}\right),
\end{aligned}
$$

and couple this operator with the spherical tensors $Q^{(8)}$ to zero angular momentum. 
There is one chiral invariant

$$
I_{6}^{\mathrm{C}}=i\left[Q^{(6)} \times\left[\partial^{(1)} \times Q^{(6)}\right]^{(6)}\right]^{(0)} .
$$

The symbol $\left[T^{(R)} \times S^{(L)}\right]^{(J)}$ denotes a spherical tensor of rank $J$, of components

$$
\left[T^{(K)} \times S^{(L)}\right]_{M}^{(J)}=T_{q}^{(K)} S_{m}^{(L)}\langle K L q m \mid J M\rangle,
$$

which is Clebsch-Gordon coupled from the spherical tensors $T^{(K)}$ and $S^{(L)}$.

Using the Wigner-Eckart theorem for the matrix elements of the angular momentum operators $J_{1}, J_{2}, J_{3}$, we can show that the chiral term of (38) equals $2 \sqrt{546} I_{6}^{\mathrm{C}}$.

Note that $Q_{m}^{(l)}$ are components of the spherical tensor

$$
Q^{(l)}=\sum_{m=-l}^{l} Q_{m}^{(l)}|l m\rangle,
$$

and that the transition law from Cartesian components to spherical components is just the complex conjugate of the transition law from Cartesian basis tensors to spherical basis tensors. For example, for $l=2$ we obtain

$$
Q_{2}^{(2)}=-\frac{1}{2}\left\{\left(Q_{11}-Q_{22}\right)-i\left(Q_{12}+Q_{21}\right)\right\}
$$

whereas the basis vector $|22\rangle$ is

$$
|22\rangle=-\frac{1}{.2}\left\{\left(\hat{\boldsymbol{e}}_{1} \otimes \hat{\boldsymbol{e}}_{1}-\hat{\boldsymbol{e}}_{2} \otimes \hat{\boldsymbol{e}}_{2}\right)+i\left(\hat{\boldsymbol{e}}_{1} \otimes \hat{\boldsymbol{e}}_{2}+\hat{\boldsymbol{e}}_{2} \otimes \hat{\boldsymbol{e}}_{1}\right)\right\}
$$

In angular momentum coupling, the derivative and angular momentum operators have to be treated like tensor components. Therefore, in (35) we have chosen the transformation law as for vector components, and also in applying the Wigner-Eckart theorem one has to be aware of this situation.

There are three positive definite terms quadratic in the gradient of $Q^{(6)}$,

$$
I_{6}^{\mathrm{G}, L}=\left[\left[\partial^{(1)} \times Q^{(6)}\right]^{(L)} \times\left[\partial^{(1)} \times Q^{(6)}\right]^{(L)}\right]^{(0)}
$$

for $L=5,6,7$.

The invariant in the one-constant approximation (37), which is quadratic in the gradient of $Q^{(6)}$, is the following linear combination of invariants (45)

$$
\left(\partial_{k} Q^{(6)}\right)_{m}^{*}\left(\partial_{k} Q^{(6)}\right)_{m}=\sqrt{11} I_{6}^{\mathrm{G}, 5}+\sqrt{13} I_{6}^{\mathrm{G}, 6}+\sqrt{15} I_{6}^{\mathrm{G}, 7}
$$

\section{Prospects}

To draw phase diagrams in Landau-Ginzburg theory amounts to determine in parameter space of the bulk and elastic free-energy those lines, planes or hyperplanes, where the symmetry of the minimizing order parameter field is changing.

Usually the order parameter fields are expanded into plane waves with helicity

$$
Q^{(l)}(\boldsymbol{x})=\sum_{\boldsymbol{k}, m} Q_{m}^{(l)}(\boldsymbol{k})|l m\rangle_{\boldsymbol{k}} \mathrm{e}^{i\left(\boldsymbol{k} \cdot \boldsymbol{x}+\gamma_{m}(\boldsymbol{k})\right)},
$$

where $|l m\rangle_{\boldsymbol{k}}$ is a spherical basis tensor with $\boldsymbol{k}$ as quantization axis. The selection of wave vectors $\boldsymbol{k}$ and phases $\gamma_{m}(\boldsymbol{k})$ fixes the symmetry of the phase. For chiral nematic liquid crystals (without external field), where $l=2$, essentially three types of wave vector sets have been used:

a) A single wave vector. The corresponding order parameter field is the single uniaxial (16) or biaxial (19) spiral or an optimum mixture of both. This field describes the cholesteric phase.

b) Wave vectors out of a cubic reciprocal lattice. These enter in description of the cubic Blue Phases [16]. 
c) Icosahedral wave vector sets, whose elements point from the centre of an icosahedron to either its vertices, faces or edges. These have been applied in models for the Blue Fog [20].

The same wave vector sets can now serve to analyse phases in metals and metallic alloys, where $l=2$ has to be replaced by $l==6$.

Set a) might describe the monoperiodic T-phase, set b) cubic Frank-Casper phases, and set c) the icosahedral quasi-crystalline phases.

The question remains unanswered, however, which type of order parameter field is describing the long-range disorder of a metallic glass.

\section{Appendix}

The starting point for an analysis of the integrity basis is a generating function, $F_{l}(q)$, for $\mathrm{O}(3)$-invariants contained in the symmetric product of an arbitrary number of identical $\mathrm{O}(3)$-tensors. For $l=6, F_{6}(q)$ is given by [31, 33]

$$
F_{6}(q) \stackrel{|q|<1}{=}-\frac{1}{2(1-q)} \sum_{\substack{\text { over residua } \\ \text { inside circle }|z|<1}}\left\{\frac{z^{20}(z-1)^{2}}{\prod_{i=1}^{6}\left(z^{i}-q\right)\left(1-z^{i} q\right)}\right\} .
$$

Table 1

Numerical values of the coefficients $a_{n}(i)$, (A2). Values of $s_{n}=\sum_{i=1}^{6} a_{n}(i)$ can directly be eompared with the results of Bystricki et al. [33]

\begin{tabular}{rlllllll}
\hline$n$ & $a_{n}(1)$ & $a_{n}(2)$ & $a_{n}(3)$ & \multicolumn{1}{c}{$a_{n}(4)$} & $a_{n}(5)$ & $a_{n}(6)$ & $s_{n}$ \\
\hline 0 & 0 & 0 & 0 & 0 & $1 / 2$ & $1 / 2$ & 1 \\
1 & 0 & 0 & 0 & -1 & 1 & 0 & 0 \\
2 & 0 & 0 & 0 & -4 & $11 / 2$ & $-3 / 2$ & 0 \\
3 & 0 & 0 & 2 & $-25 / 2$ & $35 / 2$ & -7 & 0 \\
4 & 0 & 0 & $11 / 2$ & -34 & 50 & $-41 / 2$ & 1 \\
5 & 0 & 0 & $33 / 2$ & -81 & $231 / 2$ & -50 & 1 \\
6 & 0 & -1 & $71 / 2$ & $-347 / 2$ & 250 & -108 & 3 \\
7 & 0 & $-5 / 2$ & 76 & -341 & 483 & $-423 / 2$ & 4 \\
8 & 0 & $-11 / 2$ & $297 / 2$ & -624 & $1763 / 2$ & $-769 / 2$ & 7 \\
9 & 0 & -11 & $499 / 2$ & $-2143 / 2$ & 1498 & -656 & 9 \\
10 & 0 & $-39 / 2$ & 408 & -1744 & 2432 & $-2119 / 2$ & 17 \\
11 & 0 & -32 & 648 & -2707 & $7491 / 2$ & $-3267 / 2$ & 21 \\
12 & 0 & $-101 / 2$ & 967 & $-8049 / 2$ & $11115 / 2$ & $-4827 / 2$ & 36 \\
13 & 0 & -75 & $2807 / 2$ & $-11515 / 2$ & $15815 / 2$ & $-6867 / 2$ & 45 \\
14 & $1 / 2$ & $-213 / 2$ & $3885 / 2$ & $-15899 / 2$ & $21789 / 2$ & $-9433 / 2$ & 65 \\
15 & 0 & $-293 / 2$ & $5241 / 2$ & -10619 & $28997 / 2$ & $-12545 / 2$ & 81 \\
16 & $1 / 2$ & $-387 / 2$ & $6801 / 2$ & $-27513 / 2$ & 18753 & -8094 & 110 \\
17 & $1 / 2$ & -247 & 4309 & $-34623 / 2$ & 23530 & -10150 & 131 \\
18 & 1 & -307 & $10561 / 2$ & -21193 & 28776 & $-24779 / 2$ & 168 \\
19 & 1 & -370 & 6329 & -25272 & 34241 & -14736 & 193 \\
20 & $3 / 2$ & $-867 / 2$ & $14723 / 2$ & $-58767 / 2$ & 39782 & -17096 & 232 \\
21 & $3 / 2$ & -496 & $16767 / 2$ & -33337 & 45066 & -19362 & 256 \\
22 & 2 & $-1105 / 2$ & 9286 & -36933 & 49910 & $-42839 / 2$ & 293 \\
23 & 2 & -600 & 10079 & $-79953 / 2$ & 53961 & $-46317 / 2$ & 307 \\
24 & $5 / 2$ & -637 & $21309 / 2$ & $-84579 / 2$ & $114171 / 2$ & -24480 & 336 \\
25 & 2 & -660 & 11041 & $-87473 / 2$ & 58998 & $-50611 / 2$ & 339 \\
26 & $3 / 2$ & $-667 / 2$ & $22301 / 4$ & $-44230 / 2$ & $119363 / 4$ & $-25587 / 2$ & $351 / 2$ \\
\hline & & -6 & - & & &
\end{tabular}


Direct calculations of $F_{6}(q)$ from (A1) are extremely tedious and practically impossible to perform without using some computer programs. A simpler method is to use recursive techniques [33]. The results for $L=6$ such of recursive calculations are given in [33]. As the final results are rather complicated we recalculated $F_{6}(q)$ using (A1) and the computer symbol manipulator MACSYMA. The results can be written as

$$
\begin{aligned}
& F_{6}(q)=\frac{\sum_{n=0}^{26}\left\{\sum_{i=1}^{6} a_{n}(i)\right\}\left(q^{n}+q^{52-n}\right)}{\prod_{j=2}^{11}\left(1-q^{3}\right)}=\sum_{m=0}^{\infty} u_{m} q^{m}= \\
& =1+q^{2}+q^{3}+3 q^{4}+3 q^{5}+8 q^{6}+10 q^{7}+20 q^{8}+28 q^{9}+52 q^{10}+\ldots,
\end{aligned}
$$

where coefficients, $a_{n}(i)$, are listed in Table 1. Set $\left\{a_{n}(I)\right\}$, for fixed $I$, represents the contribution to $F_{6}(q)$ from all residua in a complex plane, $z$, fulfilling the equation $z^{I}=q$.

Equation (A2) determines the integrity basis for $l=6$ while the power series expansion (A3) provides the number of linearly independent six-harmonics of degree $m$ as the expansion coefficient $u_{m}$.

\section{References}

[1] J. D. Bernal, Proc. Roy. Soc. A280, 299 (1964).

[2] F. C. Frank, Proc. Roy. Soc. A215, 43 (1952).

[3] P. J. Steinhardt, D. R. Nelson, and M. Ronchetti, Phys. Rev. B 28, 784 (1983).

[4] F. C. Frank and J. S. KASPER, Acta cryst. 11, 184 (1958); 12, 483 (1959).

[5] D. R. Nelson, Phys. Rev. B 28, 5515 (1983).

D. R. Nklson and M. Wmom, Nuclear Phys. B240, 113 (1984).

[6] P. Shechtman, I. Blech, D. Gratias, and J. W. Cahn, Phys. Rev. Lett. 53, 1951 (1984).

[7] R. Penrose, Math. Intelligencer 2, 32 (1979).

[8] D. Levine and P. J. Steinhardt, Phys. Rev. Letters 53, 2477 (1984); Phys. Rev. B 34, 596 (1986).

[9] P. Kramer and R. Neri, Acta cryst. A40, 580 (1984).

[10] L. Benderskx, Phys. Rev. Letters 55, 1461 (1985).

[11] R. L.̈̈CK, Z. phys. Chem., in the press.

[12] F. Spatepen, in: Physics of Defects, Les Houches Session XXXV, Ed. R. Balian, M. KltMAN, and J.-P. Poirier, North-Holland Publ. Co., 1981 (p. 133).

[13] P. G. De Gennes, The Physies of Liquid Crystals, Clarendon Press, Oxford 1975.

[14] H. Stegemeyer, Th. Blümel, K. Hiltrop, H. Onusseit, and F. Porsch, Liquid Cryst. 1, $3(1986)$.

[15] H. Kleinert and K. Makx, Fortschr. Phys. 29, 219 (1981).

[16] H. Grebie, R. M. Hormreich, and S. Shtrikman, Phys. Rev. A 28, 1114 (1983); A 30, 3264 (1984).

[17] S. Meiboom, M. Sammon, and W. F. Brinkman, Phys. Rev. A 27, 438 (1983).

[18] H. ONusSeit and H. Steghme Yer, Z. Naturf. 34a, 1083 (1981).

[19] M. Marcus, J. Physique 42, 61 (1981).

[20] R. M. HorNreich and S. Shtrikmax, Phys. Rev. Letters 56, 1723 (1986).

[21] D. S. Rokhsar and J. P. Sethna, Phys. Rev. Letters 56, 1727 (1986).

[22] J. P. Sethna, D. C. Wright, and N. D. Mermin, Phys. Rev. Letters 51, 467 (1983).

[23] A. R. Edmonds, Angular Momentum in Quantum Mechanics, Princeton University Press, 1957.

[24] S. Chandrasekhar, Liquid Crystals, Cambridge University Press, 1980.

[25] J. D. Bernat, Sc. Amer 203, 2, 124 (Aug. 1960).

[26] H. S. M. Coxeter, Introduction to Geometry, Wiley, New York 1969. 
[27] J. F. SADOC, J. Physique 41, C8-326 (1980).

J. F. SAdoc and R. Mosseri, Phil. Mag. B45, 467 (1982).

M. KlÉman and J. F. SA Doc, J. Physique Lettres 40, L569 (1979).

[28] K. Urban, N. Moser, and H. KronmülLER, phys. stat. sol. (a) 91, 411 (1985).

[29] M. V. JARIć, Nuclear Phys. B265, 647 (1986).

[30] B. SAlzGEBer, Diploma Thesis, University of Stuttgart 1987.

[31] B. R. Judd, W. Miller, Jr., J. Patera, and P. Winternitz, J. math. Phys. 15, 1787 (1974).

[32] R. Gaskell, A. Peccia, and R. T. Sharp, J. math. Phys. 19, 727 (1978).

[33] J. Bystricky, R. Gaskeli, J. Patera, and R. T. Sharp, J. math. Phys. 23, 1560 (1982).

[34] L. Miches, Rev. mod. Phys. 52, 617 (1980).

[35] H.-R. Trebin, Adv. Phys. 31, 195 (1982).

(Received September 1, 1987) 